\title{
PHRYNICHOS KOMMT VOR GERICHT
}

\author{
F. X. Ryan
}

\begin{abstract}
Since Herodotos says that the playwright Phrynichos was fined by "the Athenians," historians have often inferred that he was condemned by a popular tribunal. The context of the statement shows however that this formulation of Herodotos is of no avail in identifying the authority which meted out justice to Phrynichos. The amount of the fine and the outlawry of the play are better indications of popular involvement in the affair.
\end{abstract}

Nach Herodot waren die Athener bestürzt über die Eroberung Milets. Darum sei der Tragödiendichter Phrynichos, der dieses Ereignis als Verfasser und Chorlehrer the-

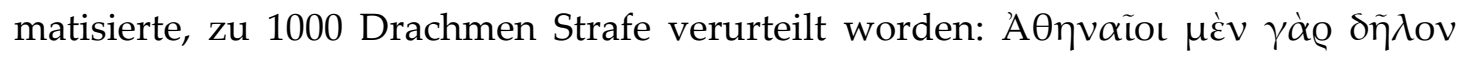

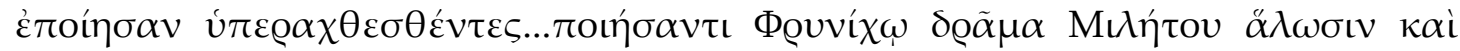

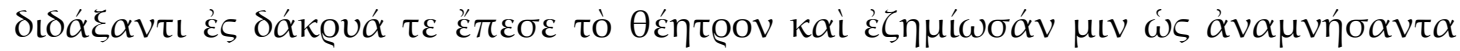

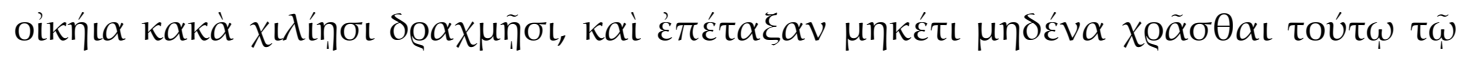

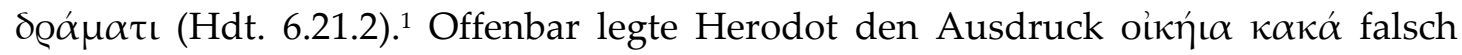
aus2: Das eigene Unglück, an das Phrynichos seine Mitbürger erinnerte, war nicht die Einnahme Milets durch die Perser im Jahre 494 v. Chr., sondern die Verwüstung Athens durch dieselben im Jahre 480, wie erst J. Roisman erkannte. ${ }^{3}$ Der Prozeß

\footnotetext{
${ }^{1}$ Die Höhe der Geldstrafe wird auch von Strab. 14.1.7 angegeben; Ammian. 28.1.4 redet zwar allgemeiner von einer Verurteilung (damnatus est), begründet aber dieselbe: quae pertulerat amabilis civitas, nullis auctorum adminiculis fulta, hos quoque dolores scaenicis adnumerasse fabulis insolenter. Ammian biete, so Marx (1928: 343), "Eigenartiges und Wesentliches, nicht nur Rhetorik“; dagegen meinte v. Blumenthal (1941: 914), „daß Herodot für das Altertum wie für uns die einzige Quelle war, “ Ammian mithin den Herodotbericht ausschmückte. Das Motiv der Athener ist bei Ammian vielleicht verständlicher, aber ein anderes als bei Herodot. Für diesen ist die Verurteilung des Phrynichos ein Beweis für das Mitgefühl der Athener, nicht für irgendwelche Schuldgefühle.

2 Der Ausdruck ist bei Herodot sonst sowohl im Singular (1.45.2) als auch im Plural (Hdt. 3.14.10, 7.152.2) belegt, und „since the phrase...has a definite Herodotean ring, it probably does not record the Athenian charge against Phrynichos“ (Rosenbloom 1993: 164); der Ausdruck ist jedoch das bzw. eine akzeptable Wiedergabe dessen, was ihm gesagt und von ihm dann mißverstanden wurde.

${ }^{3}$ Den Versuch, das Drama über das Partizip herabzudatieren, wies Ostwald (1986: 28 A. 106) zurück, denn " $\dot{\alpha} v \alpha \mu \nu \eta ́ \sigma \alpha v \tau \alpha$ does not necessarily presuppose a previous forgetting." Roisman (1988: 17-18) brachte Argumente für "the translation of the word oikeia as 'own'” vor und kam zu dem Ergebnis: "Hence Phrynichos reminded the Athenians of their own kaka and not of those of the Milesians." Dagegen könnte man einwenden, daß Herodot offenbar meinte, die Athener hätten das milesische Unglück als kein fremdes betrachtet. Trotzdem läßt sich Roismans Rekonstruktion untermauern. Wenn in Athen jedes milesische Unglück mit einem athenischen gleichgesetzt worden wäre, dann hätte Phrynichos, der ja selbst Athener und damals ein erfahrener Tragödiendichter (Suda, s. n.: Tragödiensieg schon in den J. 511-508 v. Chr.) war, dies wissen müssen und die Finger davon lassen sollen. Auch die heftige Reaktion der Zuschauer legt nahe, daß sie an die Verwüstung ihrer Heimat erinnert worden waren (vgl. Roisman 1988: 18). Nach Marx (1928: 342) erzielte der Dichter eine solche Wirkung bei den Athenern als „den mitleidvollsten aller Hellenen.“ Ob die Melier das so gesehen hätten? Bei Herabdatierung ist es auch um die Glaubwürdigkeit der Nachricht besser bestellt. Eine Geldstrafe „for 'having re-
} 
gegen Phrynichos ist demnach nicht mehr um 493/2, sondern mit Roisman in die Zeit nach $480 / 79,{ }^{4}$ wohl in die $470 s,{ }^{5}$ zu datieren.

Die Herabdatierung des Prozesses wirkt sich aber wohl nicht auf das Verfahren aus. Die Geldstrafe war so hoch, daß der Demos sie entweder in erster Instanz verhängte oder in zweiter Instanz bestätigte, ${ }^{6}$ und Demos bedeutet in diesem Fall die Eliaia. ${ }^{7}$ Es fragt sich nur, ob die Mitwirkung des Demos bezeugt ist oder nicht. Hansen meinte ohne nähere Erläuterung, „we have no evidence about the procedure applied or the court hearing the case"; Rhodes und Ostwald ihrerseits entnahmen dem Wort AOクvaĩo die Beteiligung des Demos, ${ }^{8}$ was sich erhärten zu lassen scheint, denn bei Thu-

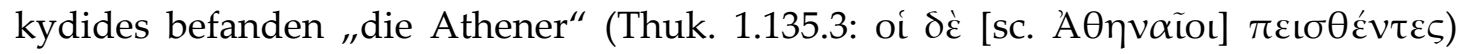
Themistokles persischer Gesinnung schuldig, und dieses Urteil wurde in einem der von Krateros aufgezeichneten psephismata gesprochen (FgrH 342 F 11a-b). Für diese Frage ist allerdings der jeweilige Zusammenhang sehr lehrreich. Bei Thukydides werden „die Athener" überzeugt, weil „die Lakedaimonier" sie überzeugen: oi $\Lambda \alpha$ -

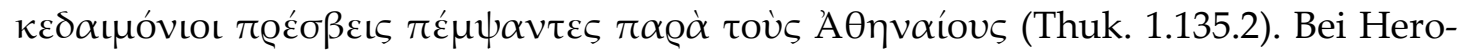
dot verhält es sich nicht anders. Die Milesier seien über das Schicksal der Sybariten betroffen gewesen, das Schicksal der Milesier habe aber die Sybariten nicht gerührt. Da Sybaris schon im Jahre 510 zerstört worden war, heißen die Sybariten hier nicht

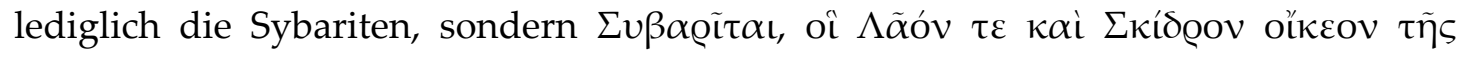

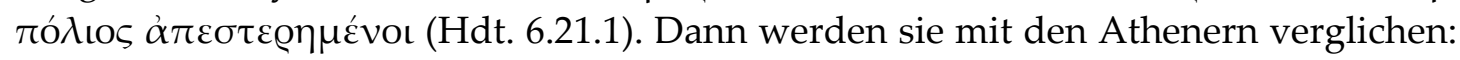

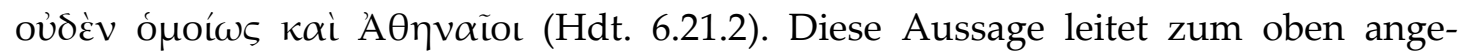
führten Bericht über Phrynichos über. Man wird also feststellen dürfen, daß es an dieser Stelle von AӨpvaĩo die Rede ist, weil Herodot sie mit den soeben erwähnten $\Sigma v \beta \alpha Q \tilde{i} \tau \alpha \iota$ vergleichen möchte. Die Nachricht, die Athener hätten Phrynichos bestraft, deckt sich also in diesem Zusammenhang mit der Nachricht, Phrynichos sei in Athen bestraft worden. Das Ethnikon verrät nicht, daß der Demos ein Urteil fällte, es kommt vielmehr einer Ortsangabe gleich.

Trotzdem kann der Fall Phrynichos der Verfassungsgeschichte viel nutzen. In seinem Bericht über den Prozeß gibt Herodot eine weitere Einzelheit preis, die in der neue-

minded the citizens of their own grief,'" so Lloyd-Jones (1966: 233), "sounds suspiciously aesthetic"; dank des späteren Aufführungsjahres ist die Anschuldigung nicht mehr verdächtig.

${ }^{4}$ Roisman (1988: 19) datierte das Stück „,after 479.“ Anderweitig ist die Vorliebe für 493/2, das angebliche Archontenjahr des Themistokles, schwächer geworden; nach Rosenbloom (1993: 172) fällt es in die Zeit „between 493/92 and the year of Marathon“; Munn (2000: 22-23) hält für möglich „any moment when the Athenians had reason to fear Persian vengeance," also die Zeit „between the end of the Ionian revolt and the arrival of Xerxes in 480.“ Unlängst ließ Pöhlmann (2001: 406) das Stück im Frühjahr 492 aufführen, allerdings ohne sich mit neuerer Literatur auseinanderzusetzen.

${ }^{5}$ Unter dem Choregen Themistokles führte er noch i. J. 476 ein Drama auf (Plut. Them. 5); vgl. v. Blumenthal (1941: 911): „Vielleicht darf man aus der Tatsache, daß 467...nicht er, sondern sein Sohn unterlag (arg. Aisch. Sept.), schließen, daß er damals tot war."

${ }^{6} \mathrm{Zu}$ einer von dieser verschiedenen Geldstrafe von 1000 Drachmen vgl. Kahrstedt (1936: 214 A. 3): Sie ist, „wie die Höhe des Betrages zeigt, natürlich ein Strafantrag vor Gericht, keine...Beamtenstrafe.“

${ }^{7}$ In dem Fluch, der zu Beginn jeder Volksversammlung ausgesprochen wurde, erwähnte der Herold neben der Boule und dem Demos auch die Eliaia (Dem. 23.97), was zeigt, daß die Eliaia eine richterliche Funktionen ausübende Volksversammlung war, zunächst also weder geloste Eliasten noch die Dikasteria da waren; vgl. Ryan 1994: 128 A. 77.

${ }^{8}$ Hansen 1980: 91, Rhodes 1979: 105, Ostwald 1986: 28-31. 
ren Forschung übergangen ${ }^{9}$ oder nur gestreift wird: Die Athener hätten auch ein Verbot des Stückes ausgesprochen. Es ist auf jeden Fall klar, daß das Verbot nicht nur auf die Wiederaufführung des Dramas zu beziehen ist. Widerlegt wurde jene Ansicht durch Marx: „Da Herodot an derselben Stelle den Dichter Phrynichus von dem Chorlehrer Phrynichus unterscheidet, durfte man auch an dieser Stelle den tref-

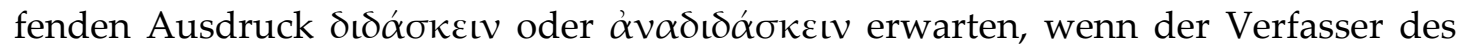
Volksbeschlusses diesen Begriff zum Ausdruck bringen wollte." Dem Infinitiv

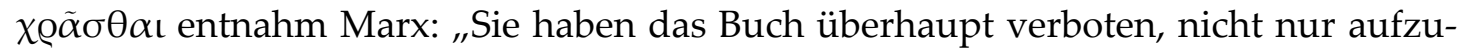
führen, sondern auch abzuschreiben, oder auch vorzulesen, im Privathaus oder auf dem Markt. "10 Auf Marx berief sich von Blumenthal, als er schrieb: Der Infinitiv bedeutet "die damnatio memoriae des Werkes."11 Aber dieser scheint das Verbot ein wenig anders verstanden zu haben als jener. Das Verbot scheint von Blumenthal mit der Vernichtung des Stückes gleichgesetzt zu haben, denn er fand es „unwahrscheinlich, daß es noch irgend jemand in der Antike hat lesen können," während Marx, der an einer Stelle tatsächlich angibt, "die Handschrift des Dichters war bald nach der ersten Aufführung vernichtet worden," an anderer Stelle bemerkt, sie sei weder beschlagnahmt noch verbrannt worden (vgl. D.L. 9.52). ${ }^{12}$ Vielleicht geht es aber bei dem Verbot weniger um die Tragödie als um die einzelnen Verse derselben. Vielleicht trägt $\chi \varrho \tilde{\alpha} \sigma \theta \alpha \mathrm{L}^{13}$ dem Rechnung, was für diese Zeit sonst bezeugt ist und im vorliegenden Fall wenigstens wohl wahrscheinlicher war als die Wiederaufführung des Stückes, nämlich Entlehnungen aus ihm. Wir erfahren etwa, daß Euripides in seiner Alkestis einige Details aus der des Phrynichos beibehielt (Serv. auct. Aen. 4.694). Man war vielleicht bestrebt, auch oder sogar vor allem diesen Entlehnungen zuvorzukommen. Doch wie dem auch immer gewesen sein mag - dies eine ist sicher: Das Verbot wurde von keinem Magistrat erlassen.

Setzen wir einmal voraus, was nicht unwahrscheinlich ist, daß die beiden Entscheidungen in ein und derselben Quelle stehen, weil sie an ein und demselben Tag von ein und derselben Institution getroffen wurden. Auch unter dieser Voraussetzung gelangen wir zu der Ansicht, daß die Eliaia Phrynichos zur Verantwortung zog. Das Verbot war nämlich eine politische Entscheidung und das einzige Organ, das beide Entscheidungen getroffen haben könnte, war die Volksversammlung, die sowohl als Ekklesia wie auch als Eliaia ihre Aufgaben ausführte. ${ }^{14}$ Ostwald meinte zwar, "the defendant was...enjoined from any future performances of the play, ${ }^{15}$ diese Formulierung verwandelt aber die politische Entscheidung in eine gerichtliche, denn Hero-

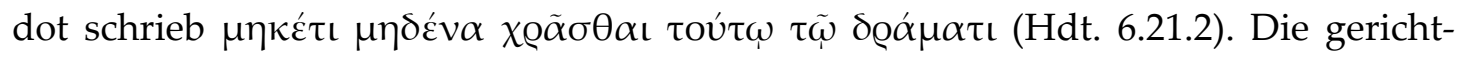
liche Entscheidung ging nur den Angeklagten an. Da es in späterer Zeit Sache eines Dikasterion war, höhere Geldstrafen zu verhängen, ist es nicht unwahrscheinlich,

\footnotetext{
${ }^{9}$ Rhodes (1979: 105) erwähnt nur die Geldstrafe, Hansen (1980: 91) erwähnt nicht einmal sie.

${ }^{10}$ Marx 1928: 346.

${ }^{11}$ v. Blumenthal 1941: 914.

12 v. Blumenthal 1941: 914; Marx 1928: 341, 346.

${ }^{13}$ Wir wissen nicht, ob genau dieses Wort im Texte des Beschlusses stand.

${ }^{14}$ Anscheinend hielt v. Wilamowitz (1907: 91 m. A. 57) die Geldstrafe für eine in einem Gesetz vorgesehene Strafe, deren Verhängung „kein richterlicher Act“ war, weil sie mit dem Verbot in einem „,besonderen Beschluß“ kundgegeben wurde.

15 Ostwald 1986: 28.
} 
daß zum Zeitpunkt der Verurteilung des Phrynichos noch keine Dikasteria vorhanden waren. Wir meinten eingangs, daß die Herabdatierung des Prozesses das Verfahren nicht betrifft, da sich Phrynichos vor der Eliaia verantworten mußte, egal ob dies schon um 493 oder erst in den 470s geschah. Die Herabdatierung des Prozesses ist aber trotzdem für die Verfassungsgeschichte von großem Belang, denn der Fluch des Ausrufers (Dem. 23.97) beweist nur, daß die Volksversammlung einst das Volksgericht bildete, während der Prozeß des Phrynichos wahrscheinlich erscheinen läßt, daß dies noch in den siebziger Jahren des fünften vorchristlichen Jahrhunderts der Fall war.

Setzt man weiterhin voraus, daß ein und dieselbe Sitzung beide Entscheidungen fällte, wird man bezweifeln, daß die von Herodot angegebene Reihenfolge stimmt. Es liegt nämlich nahe zu vermuten, daß die Ekklesia zu Beginn der Sitzung das Verbot aussprach, die Versammlungsteilnehmer dann vereidigt wurden und als Eliasten die Geldstrafe verhängten. Es ist aber nicht von vornherein auszuschließen, daß die Sitzung mit der Vereidigung anfing. Es ließe sich denken, daß die Eliaia weder eine andere Institution war als die Ekklesia ${ }^{16}$ noch die gleiche Institution mit anderen Befugnissen, sondern die gleiche Institution mit weiteren Befugnissen. Man könnte meinen, daß auch die politische Entscheidung von der Eliaia getroffen worden wäre, weil sie eine mit richterlicher Gewalt ausgestattete Ekklesia dargestellt hätte. Die spätere Probole ${ }^{17}$ läßt aber vermuten, daß die Eliaia erst auf die Ekklesia folgte, denn später ging ein Volksbeschluß dem eigentlichen Prozeß voraus. ${ }^{18}$ Schon die Existenz eines Richtereides verrät, daß die Richtertätigkeit als eine besondere betrachtet wurde, was wahrscheinlich macht, daß die Vereidigung der Richter mit dem Richten aufs engste verquickt war. Demnach hat Herodot bzw. sein Gewährsmann die Reihenfolge der Entscheidungen wohl umgekehrt. Mitgeteilt wurde zunächst die Entscheidung, die allein Phrynichos betraf, und erst danach diejenige, die auch anderen galt. Das Verbot war mithin zugleich ein Präjudiz des Volkes zuungunsten des Phrynichos.

\author{
Dr. Francis X. Ryan \\ Technische Universität Dresden, Institut für Geschichte \\ E-mail:fxryan@gmx.de
}

\footnotetext{
${ }^{16}$ Solon hielt die politischen und richterlichen Funktionen des Demos nicht so auseinander wie wir es tun und die Athener ebenfalls wohl noch in der archaischen Zeit es taten, weswegen die Bezeichnung Eliaia erst in der nachsolonischen Zeit entstehen konnte; Solon selbst erkannte hier also nur eine einzige Institution. Siehe Ryan 2003: 1-4.

${ }^{17}$ Das Gesetz, das die Probole für die Störung der großen Dionysien zuließ (Dem. 21.8-9) und möglicherweise dieses Verfahren erst einführte (so MacDowell 1990: 14), war noch nicht in Kraft, als Alkibiades Chorege war (Dem. 21.147). Historiker, die diese Stelle des Demostehens nicht kannten, wie noch G. Freymuth (1955: 56 A. 2), ließen die Athener den Dichter in der Klageform der Probole zur Rechenschaft ziehen; wer die Stelle des Demosthenes kennt, wie Rosenbloom (1993: 161 A. 5), der bestreitet, daß die Athener gegen Phrynichos in der Form der Probole vorgingen. Das Verfahren der Probole könnte aber älter sein als das von Demosthenes angeführte Gesetz, das die Volksversammlung im Theater nach den städtischen Dionysien vorschrieb, bei welcher die Probole auf der Tagesordnung stand (Dem. 21.8; vgl. A.P. 43.5). Eine Vorabstimmung des Volkes, ob sie denn Probole hieß oder nicht, wäre sicher vor der Verabschiedung des fraglichen Gesetzes stattthaft gewesen.

18 Vgl. MacDowell (1990: 16): „It has sometimes been supposed that the name probole was applicable only to the hearing in the Ekklesia.... That is a misapprehension. Several kinds of legal action in Athens were named from the manner in which they were initiated."
} 


\section{Bibliographie}

Blumenthal, A. von (1941) ,Phrynichos 4.' - RE 20, 911-17.

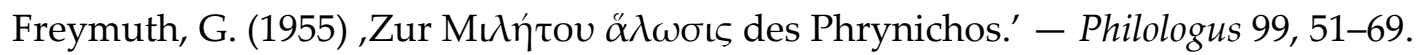

Hansen, M. H. (1980) ,Eisangelia in Athens: A Reply.' - JHS 100, 89-95.

Kahrstedt, U. (1936) Untersuchungen zur Magistratur in Athen. Stuttgart: W. Kohlhammer.

Lloyd-Jones, H. (1990) ,Problems of Early Greek Tragedy: Pratinas and Phrynichus.' - H. Lloyd-Jones, Greek Epic, Lyric, and Tragedy, Oxford: University Press [zuerst 1966], 225-237.

MacDowell, D. M. (1990) Demosthenes: Against Meidias. Oxford: University Press.

Marx, F. (1928) ,Der Tragiker Phrynichus.' - RhM 77, 337-60.

Munn, M. (2000) The School of History: Athens in the Age of Socrates. Berkeley: University of California Press.

Ostwald, M. (1986) From Popular Sovereignty to the Sovereignty of Law. Berkeley: University of California Press.

Pöhlmann, E. (2001) ,Das 'Griechische Wunder' und die Tragödie.' - Gab es das Griechische Wunder?, hg. von D. Papenfuß und V. M. Strocka. Mainz am Rhein: Philip von Zabern, 401-415.

Rhodes, P. J. (1979) ,Ei் $\alpha \gamma \gamma \varepsilon \lambda i ́ \alpha$ in Athens.' - JHS 99, 103-14.

Roisman, J. (1988) ,On Phrynichos' Sack of Miletos and Phoinissai.' - Eranos 86, 1523.

Rosenbloom, D. (1993) ,Shouting 'Fire' in a Crowded Theater: Phrynichos's Capture of Miletos and the Politics of Fear in Early Attic Tragedy.' - Philologus 137, 15996.

Ryan, F. X. (1994) ,The Original Date of the $\delta \tilde{\eta} \mu \mathrm{s} \varsigma \lambda \eta \eta \theta v \omega v \nu$ Provisions of IG I ${ }^{3} 105 . '$ - JHS 114, 120-34.

Ryan, F. X. (2003) ,The Solonian Denomination for the Eliaia.' - OsOBA 8, 1-5.

Wilamowitz-Moellendorff, U. von (1907) Einleitung in die griechische Tragödie. Berlin: Weidmannsche Buchhandlung. 